\title{
Etude De La Diversite Des Termites (Isoptera) Dans Quelques Localites De La Region De Kolda (Haute Casamance, Senegal)
}

\section{Hassoum Sane \\ Tamsire Samb}

Institut fondamental d'Afrique noire Cheikh Anta Diop, Laboratoire de

Zoologie des Invertébrés terrestres, BP 206 Dakar, Sénégal,

Laboratoire de Biologie évolutive, Ecologie et Gestion des Ecosystèmes, Département de Biologie animale, Faculté des Sciences et Techniques,

Université Ch. A. Diop, Dakar, Sénégal.

\section{Abdoulaye Baïla Ndiaye}

Institut fondamental d'Afrique noire Cheikh Anta Diop, Laboratoire de Zoologie des Invertébrés terrestres, BP 206 Dakar, Sénégal,

\section{Cheikh Tidiane Ba}

Laboratoire de Biologie évolutive, Ecologie et Gestion des Ecosystèmes, Département de Biologie animale, Faculté des Sciences et Techniques, Université Ch. A. Diop, Dakar, Sénégal.

\begin{abstract}
Termites (Isoptera) are invertebrates that play many ecological functions in terrestrial ecosystems, especially in tropical areas. Their impacts on the quality and the fertility of the soil are well known. However, in Senegal termite fauna is still poorly known. Thus, to contribute to the knowledge of termites of Senegal we have conducted a study on their diversity in the region of Kolda (Casamance). Termites were sampled in transects of $100 \mathrm{~m}$ long and $20 \mathrm{~m}$ wide. Termite workers, soldiers and some time reproductives are collected in vials filled with ethanol $70^{\circ}$. Thirty (30) termite species have been identified. Five of them are new records for Senegal. The four trophic groups xylophagous, fungus-growing termites, harvester or foraging termites and soil-feeding were represented. The fungusgrowing termites are more diversified. According to the types of nests, termites encountered are divided into 3 groups. The more diversified are those that build ground-nest without fungus.
\end{abstract}

Keywords: Diversity, Termites, Isoptera, Haute Casamance, Senegal 


\section{Résumé}

Les termites sont des invertébrés qui remplissent de nombreuses fonctions écologiques, notamment dans les écosystèmes tropicaux. Leurs impacts sur la qualité et la fertilité des sols sont bien connus. Toutefois les connaissances sur la faune termitique du Sénégal sont limitées. Ainsi, pour contribuer à la connaissance des termites du Sénégal nous avons réalisé une étude sur leur diversité dans la région de Kolda. Les termites ont été récoltés dans des transects de $100 \mathrm{~m}$ de long sur $20 \mathrm{~m}$ de large. Tous les individus rencontrés sont collectés à la pince et conservés dans des flacons contenant de l'éthanol $70^{\circ}$. Cette étude a permis de recenser 30 espèces de termites dont cinq sont nouvelles au Sénégal. Les quatre groupes trophiques à savoir les champignonnistes, les humivores, les lignivores et les fourrageurs ont été représentés. Celui des champignonnistes est le plus diversifié. Sur les quatre types de nids, trois ont été recensés. Les nids en terre sans meule à champignon sont les plus diversifiés.

Mots clés : Termites Isoptera, Diversité, Haute Casamance, Sénégal

\section{Introduction}

Les termites sont des Insectes appartenant à l'Ordre des Blattaria et à l’Infra Ordre des Isoptères (Krishna et al., 2013). Il existe à travers le monde 3106 espèces vivantes et fossiles connues (Krishna et al., 2013) réparties dans 12 familles (Engel et al., 2009; Engel, 2011; Krishna et al., 2013). Ils sont largement distribués dans le monde surtout dans les régions tropicales, subtropicales et semi-arides (Eggleton, 2000). Les savanes boisées et les forêts sont leurs domaines de prédilection mais ils peuplent aussi les steppes et les terres arides (Noirot et Alliot, 1947). Ces insectes sociaux sont surtout connus pour les dégâts qu'ils causent aux habitations et aux cultures (Zaremski et al., 2009). Ils sont considérés comme la principale source de nuisance dans les régions tropicales. A la recherche de nourriture, les termites attaquent le bois ouvré, les textiles et les papiers. Leurs effets néfastes ont été démontré sur les plantations et les arbres fruitiers (Agbogba et Roy-Noël, 1982 ; Han et Ndiaye 1996, 2006 ; Anani Kotoklo et al. 2011) sur les cultures (Han et Ndiaye, 1998 ; Akpesse et al., 2008) et sur les bâtiments (Han, 2000).

Cependant, ils ont des fonctions importantes dans les écosystèmes terrestres. Ils jouent un rôle agrologique certain qui se traduit par des modifications d'ordre mécanique et qualitatif du sol (Garba et al., 2011). L'effet des termites sur les propriétés physiques du sol est principalement exercé à travers leurs activités de fouisseurs et de constructeurs (Duboisset, 2003). En effet, ils creusent de nombreuses galeries souterraines, qui 
facilitent l'infiltration de l'eau et la circulation de l'air et des minéraux dans les sols (Jouquet et al., 2011). Sur le plan qualitatif, ils sont impliqués dans le processus de décomposition de la matière organique, la concentration, le stockage et la redistribution des constituants minéraux et organiques (Bignell et al., 1997 ; Freymann et al., 2008, Diop et al. 2013). Dans les zones de savanes arides et semi-arides, les termites constituent la macrofaune du sol la plus active pendant la saison sèche (Diop et. al., 2013). Ils sont les principaux agents de décomposition de la matière organique (Rosin \& Leponce, 2004). Cette activité enrichit le sol en éléments nutritifs (Ouédraogo et al., 2004; Jouquet et al., 2011 ; Freymann et al., 2008).

La plupart des études sur les termites au Sénégal ont concerné la presqu'île du Cap-Vert (Roy-Noël, 1974; Roy-Noël \& Wane, 1977; RoyNoël, 1982; Guèye, 1987 ; Mbao, 2015 ; Sonko, 2015), le nord (Lepage, 1974; Samb et al., 2011; Guèye, 2011) et le centre (Sarr, 1999). Peu de travaux portant sur les termites ont été réalisés à Kolda. Les seuls travaux sur les termites en Haute Casamance sont ceux de Fall et al. (2000) et Brauman et al. (2000). Ces études ont porté sur l'influence de la jachère sur les termites et l'action des termites sur des caractéristiques du sol.

Compte tenu de l'importance des termites dans le fonctionnement des écosystèmes et le peu de connaissances sur cette faune dans le Sud du Sénégal, nous nous sommes intéressés aux diversités spécifique et fonctionnelle des termites dans la région de Kolda, en Haute Casamance.

\section{Matériel et méthodes}

\section{Zone d'étude}

L’étude a été réalisée dans la région de Kolda, dans les localités suivantes (fig. 1): Wassadou (département de Vélingara), Médina Yoro Foula (département de Médina Yoro Foula) et Saré Samba Kayogo (département de Kolda).

La région de Kolda se situe entre $12^{\circ} 20$ et $13^{\circ} 40$ de latitude nord et $16^{\circ}$ de longitude ouest. Elle correspond à la Haute Casamance qui est un territoire sec recouvert par une forêt claire (Seck, 1955). Le climat est de type soudanien avec deux saisons distinctes : une saison sèche de 7 mois (de novembre à mai) et une saison pluvieuse de 5 mois (de juin à octobre). Dans cette zone la pluviométrie varie d'une année à l'autre. D’après les données de l'Agence nationale de la Météorologie et de l'Aviation civile (ANACIM), les cumuls annuels des pluies de 2010 à 2013 enregistrées au niveau de la région de Kolda sont respectivement 1353,4 mm, 871,6 mm, 1242,5 mm et $798,9 \mathrm{~mm}$. Les températures moyennes mensuelles ont oscillé entre $25,2^{\circ} \mathrm{C}$ pour le mois le plus frais (janvier) et $33,5^{\circ} \mathrm{C}$ pour le mois le plus chaud (mai). Les sols, essentiellement ferrugineux, recouvrent une cuirasse latéritique qui affleure par endroits (Niane, 1984). La végétation est marquée 
par des peuplements à affinité soudano-guinéenne qui s’éclaircissent au fur et à mesure qu'on progresse vers l'Est. Quatre espèces, Bombax costatum, Pterocarpus erinaceus, Daniellia oliveri et Cordyla pinnata, prédominent dans la strate arborée avec un sous-bois composé de Combrétacées. Le tapis herbacé, consistant, est essentiellement composé de graminées.

\section{Méthodes}

Les échantillonnages de termites ont été réalisés à Wassadou, Médina Yoro Foula et Saré Samba Kayogo. À Wassadou, les stations échantillonnées sont trois (3) champs en jachère, un (1) verger et deux (2) stations en savane boisée. À Médina Yoro Foula, ce sont deux (2) stations en savane boisée et une (1) station dans une rizière qui ont été échantillonnées. Pour Saré Samba Kayogo, les échantillonnages ont porté sur deux (2) stations en savanes boisées et une (1) station dans une savane anthropisée en bordure d'un site de production maraichère.

Au niveau de chaque station, les termites sont recherchés, dans un transects de 100 m X 20 m, dans le sol, dans la litière, sur les arbres (écorces, troncs, branches, racines), sur le bois mort, les souches et dans les termitières. Les soldats et les ouvriers sont prélevés ainsi que les individus des autres castes (roi, reine, essaimants) s’ils sont rencontrés. Les termites récoltés sont conservés dans des tubes contenant de l'éthanol $70^{\circ}$ puis transportés au laboratoire pour identification et mise en collection.

L’identification des espèces a été faite au laboratoire de Zoologie des Invertébrés terrestres de l'Institut fondamental d'Afrique noire Cheikh Anta Diop (IFAN-Ch. A. Diop). Les échantillons ont été observés et photographiés à l'aide d'une loupe binoculaire équipée d'une caméra reliée à un ordinateur. Nos spécimens ont été comparés aux spécimens de référence de la collection de l'IFAN directement sous la loupe et/ou à l'ordinateur à partir des images prises avec une caméra. Les travaux de Silvestri (1914-15), Sjöstedt (1925), Emerson (1928), Grassé (1937, 1944-45), Bouillon et Mathot (1965, 1971) et Ndiaye (2014) ont également été utilisées comme référence dans l'identification des espèces.

Tous les spécimens collectés et étudiés sont en conservation dans la collection du Laboratoire de Zoologie des Invertébrés terrestres de l'IFAN (Université Cheikh Anta Diop, Dakar, Sénégal).

\section{Traitement des données \\ Groupes fonctionnels}

Les différentes espèces de termites identifiées ont été classées en fonction du régime alimentaire et du type de nid. En fonction de la qualité du bois et/ou du mode de récolte, les termites sont classés en i) lignivores, ii) humivores, iii) champignonnistes et iv) fourrageurs. 
Chez les Termites, il existe une grande diversité de nids qui peuvent se distinguer notamment par leur position dans le milieu, leur matériau de construction, leur taille, leur morphologie externe et leur structure interne (Grassé, 1984 ; Deligne \& De Coninck, 2010). Ainsi, les termites ont été classés en i) nids creusés dans le bois, ii) nids en carton stercoral, iii) nids en terre sans meules à champignon et iv) nids en terre et à meules à champignons.

\section{Analyses statistiques}

Les données ont été analysées avec le logiciel XLSTAT 6.1.9. La répartition des groupes trophiques suivant les biotopes étudiés a été soumise à un test de khi-2 au seuil de $5 \%$. Les données sur la répartition des espèces ont été soumises à une analyse factorielle des correspondances (AFC) à partir d'un tableau croisé biotopes - groupes trophiques et biotopes-types de nids prenant en compte la présence ou l'absence de groupe trophique ou d'un type de nid dans un biotope.

\section{Résultats}

\section{Diversité spécifique}

\section{Diversité spécifique globale}

Nous avons identifié 30 espèces de termites pour l'ensemble des 12 stations. Les espèces se répartissent dans deux familles, 5 sous-familles et 14 genres. Elles sont présentées dans la liste ci-dessous en suivant l'ordre systématique proposé par Krishna et al. (2013).

Famille RHINOTERMITIDAE Froggatt, 1897

Sous famille COPTOTERMITINAE Holmgren, 1910 (1 genre)

Genre Coptotermes Wasmann, 1896 (1)

Famille TERMITIDAE Latreille, 1802 (5 sous-familles)

Sous-famille Macrotermitinae Kemner, 1934 (4 genres)

Genre Ancistrotermes Silvestri, 1912 (2 espèces)

Genre Macrotermes Holmgren, 1909 (2 espèces)

Genre Microtermes Wasmann, 1902 (3 espèces)

Genre Odontotermes Holmgren, 1910 (5 espèces)

Sous-famille NASUTITERMITINAE Hare, 1937 (2 genres)

Genre Fulleritermes Coaton 1962 (1 espèce)

Genre Trinervitermes Holmgren, 1912 (4 espèce)

\section{Sous-famille CUBITERMITINAE Weidner, 1956 (3 genres)}

Genre Cubitermes Wasmann, 1906 (4 espèces)

Genre Noditermes Sjostedt, 1924 (1 espèce)

Genre Tuberculitermes Holmgren 1912 (1 espèce).

Sous-famille TERMITINAE Latreille, 1802 (4 genres)

Genre Amitermes Silvestri, 1901 (1 espèce) 
Genre Microcerotermes Silvestri, 1901 (3 espèces)

Genre Pericapritermes Silvestri, 1914 (1 espèce)

Genre Promirotermes Silvestri, 1914 (1 espèce).

\section{Diversité spécifique par localité}

\section{Diversité spécifique à Médina Yoro Foula (Tableau I)}

Dans cette localité deux biotopes ont été échantillonnés : la savane boisée et la rizière. Nous avons recensé 19 espèces de termites. Les termites ont été récoltés dans le bois mort, sur des arbres morts et vivants, dans des nids, dans le sol et sur de la bouse de vache sèche. La diversité est plus importante dans la savane boisée avec 17 espèces de termites. Au niveau de la rizière, 6 espèces ont été rencontrées.

\section{Diversité spécifique à Saré Samba Kayogo (Tableau II)}

Les termites ont été récoltés dans des nids, sur du bois mort, sur des arbres et arbustes morts et vivants, sur de la litière, sur de la bouse de vache sèche, sur des palmiers au niveau d'une savane boisée et d'une palmeraie anthropisée. Nous avons identifié 15 espèces de termites. La diversité spécifique est plus importante dans la savane boisée avec 11 espèces sur les 15 rencontrées dans la localité.

\section{Diversité spécifique à Wassadou (Tableau III)}

À Wassadou, 4 biotopes ont été explorés : cuvette inondable, champs et jachères, vergers et savane boisée. Nous avons identifié 21 espèces de termites. La diversité spécifique est plus importante dans les vergers avec 15 espèces sur les 22 recensées. Les espèces ont été prélevées dans des nids, sur des arbres, sur du bois mort, sur de la bouse de vache sèche et sur des souches d'arbres et d'arbustes.

\section{Diversité fonctionnelle}

\section{Diversité selon le groupe trophique (Tableau IV)}

Les 4 groupes trophiques sont représentés sur la liste des espèces inventoriées. Les champignonnistes (12 espèces) et les humivores (8 espèces) sont les plus diversifiés. Les lignivores et les fourrageurs, avec respectivement 6 et 4 espèces, sont les moins diversifiés.

La figure 2 représente l'analyse factorielle des correspondances qui croise les six biotopes et les 4 groupes trophiques. L'axe F1 et l'axe F2 représentent respectivement $64,75 \%$ et $29,45 \%$ de l'inertie totale. L'interprétation des données se fera sur l'axe F1 qui porte la majorité des informations.

Le test du khi-2 au seuil de 0,05 montre une liaison significative entre les biotopes et les groupes trophiques avec une p-value $<0,0001$. 
La carte factorielle permet de constater que les champignonnistes sont associés à la savane boisée, à la palmeraie et à la cuvette inondable. Les lignivores sont associés à la savane boisée. Les fourrageurs sont associés à la rizière et les humivores aux vergers.

\section{Diversité selon les nids}

Au total 70 nids appartenant à trois types de nids (nids en carton stercoral, nids en terre sans meule à champignon, nids en terre et à meule à champignon) ont été recensés dans les 6 types de biotopes (champs, cuvette, palmeraie, rizière savane boisée, vergers). Signalons que les espèces à nids creusés dans le bois sont absentes dans nos récoltes. Nous n'avons pas observé de nid arboricole. Cependant une espèce à nid arboricole (Microcerotermes fuscotibialis) a été récoltée à Wassadou.

Les deux axes de l'analyse factorielle des correspondances représentent respectivement 96,66\% et 3,34\% de l'inertie totale (Fig. 3). La carte factorielle permet de constater que les nids en carton stercoral et les nids en terre et à meule à champignon sont associés à la savane boisée et aux vergers. Les nids en terre sans meule à champignon sont associés aux vergers et aux champs. L'accolement du point représentant les nids en terre sans meule à champignon à l'axe F1 indique qu'ils sont les plus abondants.

\section{Discussion}

Au total, 30 espèces de Termites réparties dans 14 genres, 5 sousfamilles (Coptotermitinae, Macrotermitinae, Termitinae, Cubitermitinae, Nasutitermitinae) et 2 familles (Rhinotermitidae et Termitidae) ont été recensées. Des études faites auparavant en Casamance par Fall et al. (2000) et Ndiaye \& Han (2002) ont permis de recenser respectivement 18 et 23 espèces de Termites. Ces différences s'expliqueraient par le fait que nous avons travaillé dans des biotopes plus diversifiés tandis que ces auteurs ont prospecté des jachères et des vergers.

Les inventaires réalisés au nord du Sénégal à Widou (Ndiaye et Samb, 2012), au Ferlo (Lepage, 1974), à Kaolack (Sarr, 1999) ont permis le recensement respectif de 16, 23 et 20 espèces de Termites. Ces différences avec nos résultats s'expliqueraient par le fait que selon (Eggleton, 2000), la répartition des termites se traduit par une diversité et une abondance qui varient d'un écosystème à un autre en fonction du climat, du type de sol et de la végétation. Dans une revue de la diversité des termites au Sénégal, Sarr et al. (2005) avaient constaté que la diversité et l'abondance des termites baissaient du sud (climat soudano-guinéen) au nord (climat sahélien).

La diversité spécifique plus importante dans les vergers que dans les savanes et les champs serait due au fait que ces vergers d'anacardiers et de manguiers sont situés sur des sites humides et protégés des feux de brousse, 
fréquents dans la région, qui réduisent la diversité des termites (Roy-Noël 1978). Toutefois, de façon générale la diversité spécifique est plus faible dans les zones les plus anthropisées. Ceci est en accord avec les travaux de Samb et al. (2011) qui avaient observé que la diversité des espèces diminuait dans les zones les plus fréquentées par les humains.

Toutes ces espèces ont été antérieurement signalées au Sénégal à l'exception de Cubitermes sp. 1, Cubitermes sp. 2, Odontotermes erraticus, Odontotermes sp. 1 et Odontotermes sp. 2 qui sont nouvelles pour le Sénégal.

Odontotermes erraticus est décrite du Niger (Grassé, 1944-45) où il a été trouvé sur du bois, sur des excréments d’herbivores, sur du papier et autres objets de campement pourvus de cellulose. Odontotermes sp. 1 présente des antennes composées de 17 articles. L’article III est le plus court. Les bords latéraux de la tête convergent faiblement en avant. La plus grande largeur de la tête se situe vers l'arrière. Les mandibules sont droites et recourbées à leur extrémité. La mandibule gauche possède une dent marginale nette.

Sur le plan trophique, la présence des 4 groupes traduit une bonne diversité fonctionnelle de la faune termitologique. Le peuplement est dominé par les champignonnistes. Ce résultat est en accord avec les travaux de Tra Bi et al. (2010) qui expliquent la dominance des champignonnistes par leur adaptation favorisée par la relation symbiotique qu'ils entretiennent avec le champignon Termitomyces qui leur facilite la dégradation du bois.

L'attachement des champignonnistes et des lignivores à la savane boisée serait due à l'abondante de nourriture en bois et litière que ce biotope leur procure. En effet, les champignonnistes récoltent leur nourriture à partir de feuilles mortes, de bois morts et d'herbe desséchées. Quant aux lignivores, ils se nourrissent de bois vivant, morts secs ou en décomposition. La présence de sol argileux dans la palmeraie et la rizière facilite l'implantation des champignonnistes dans ces biotopes. Dans les vergers, l'humidité élevée et l'abondance de la matière organique provenant des feuilles des manguiers et des anacardiers favorisent l'activité des humivores qui sont des termites se nourrissant d'humus ou de sol riche en matière organique qu'ils digèrent grâce à l'aide de bactéries symbiotiques contenues dans leur tube digestif (Deligne \& De Coninck, 2010). Les fourrageurs sont des termites qui se nourrissent essentiellement d’herbes découpées en minuscules brindilles qu'ils stockent dans leur nid (Bodot, 1967). Ainsi, la rizière avec l'abondance des graminées, cultivées ou non, est un milieu propice à leur développement.

Les Termites constructeurs de nids en terre sans meule à champignon étaient plus abondants. Les effectifs les plus importants ont été obtenus dans les savanes boisées et les vergers. Ce sont les nids des espèces de termites 
des genres Cubitermes, Noditermes, Pericapritermes, Tuberculitermes, Trinervitermes construits à partir avec leurs fécès ou de la terre prélevée directement dans le milieu et imprégnée de salive (Grassé, 1984 ; Deligne \& De Coninck, 2010). Ce résultat corrobore ceux de Zaremski et Louppe (2016). Selon ces auteurs, dans les zones plus humides, où la température et l'humidité sont régulées par un couvert forestier dense, les termites ne développent que rarement des termitières cathédrales. Ils y développent plutôt des termitières en forme de champignons, ou des « nids » en matériel cartonneux faits de bois digéré, plus ou moins lié par des particules de terre au sol ou accrochés dans les arbres, ou des nids hypogés peu ou pas visibles en surface. En Côte d'Ivoire, Tano et Lepage (1990) ont enregistré les densités de nids les plus importantes pour Cubitermes spp. dans les savanes. Les champs cultivés sont caractérisés par une faible densité du fait de la destruction systématique des nids. Les densités les plus importantes des nids en terre et à meule à champignons, nd épigé de Macrotermes bellicosus, ont été enregistrées dans les savanes.

\section{Conclusion}

Cette première étude d'ensemble sur la faune de termites de la Haute Casamance a permis de recenser 33 espèces qui se répartissent dans deux familles (Rhinotermitidae et Termitidae), 5 sous-familles et 14 genres. A l'échelle des sites, il est noté une différence dans la composition des espèces suggérant une structuration des communautés sous les effets combinés des des paramètres environnementaux (types de sol, qualité et disponibilité de la nourriture, humidité...) et l'action anthropique.

\section{Remerciements}

Cette étude est réalisée dans le cadre d'un projet de recherche sur les termites de l'Afrique de l'Ouest financé par l'Union économique et monétaire ouest africaine (UEMOA).

\section{References:}

1. Agbogba C. \& Roy-Noël J. (1982). L’attaque des arbres par les termites dans la presqu'île du Cap-Vert (Sénégal). Bulletin de l'IFAN. T. 44. Sér. A, n³-4.

2. Akpesse A. A., Kouassi P. K., Tano Y. \& Lepage M. (2008). Impact des termites dans les champs paysans de riz et de mais en savane subsoudanienne (Booro-Borotou, Côte-d'Ivoire). Sciences \& Nature Vol. $5 \mathbf{N}^{\circ} 2$ : 121 - 131.

3. Anani Kotoklo E., Amévoin K., Robert A., Tano Y., RoulandLefèvre C. \& Glitho I. A. (2011). Dégâts causés par les termites sur 
les cannes à sucre au sud du Togo. Cam. Jour. Biol. Bioch. Sc., 19 : 1-10.

4. Bignell D. E., Eggleton P., Nunes L. \& Thomas K. L. (1997). Termites as mediator of carbon fluxes in tropical forest: budget for carbon dioxide and methane emission. In : Watt A. D., Stork N. E., Hunter M. D. (Eds). Forests and insects (Chapman and Hall, London). pp. 109-134.

5. Bodot P. (1967). Etude écologique des Termites des savanes de Basse Côte d’Ivoire. Insectes sociaux, Paris, Volume XIV, n³, pp. 229258.

6. Bouillon A. \& Mathot G. (1965). Quel est ce termite africain ? Zooleo. $\mathrm{N}^{\circ}$ 1. (Ed). Université de Léopoldville, Léoplodville.

7. Bouillon A. \& Mathot G. (1971). Quel est ce termite africain ? Zooleo, 1, supplément.

8. Brauman A., Fall S. \& Chotte J. L. (2000). Caractéristiques organique, physique et microbiologique du sol soumis à l'influence des termites : Étude comparative de deux espèces dominantes des sols en jachère (Haute-Casamance, Sénégal). In : La jachère en Afrique tropicale, Ch. Floret, R. Pontanier; John Libbey Eurotext, Paris, pp. 308-316.

9. Deligne J. \& De Coninck E. (2010). Les collections de nids de Termites (Isoptera) du Musée royal de l'Afrique centrale. Bulletin S.R.B.E./K.B.V.E ; 146 : 139-150.

10. Diop A., Ndiaye A. B. \& Ba Ch. T. (2013). Décompositions de la bouse de bovin sèche et macrofaune associée en zone sahélienne semi-aride (Matam, Sénégal). International Journal of Biological and Chemical Sciences, 7 (1): 147-162.

11. Duboisset A. (2003). L'importance agricole des termitières épigées dans le nord du Cameroun: l'exemple des nids de Macrotermes subhyalinus et d'Odontotermes magdalenae. Thèse en Sciences et techniques de l'environnement. Paris : Université de Paris-Val-deMarne, 2003, 479 p.

12. Eggleton P. (2000). Global patters of termite diversity. In Termites: Evolution, Sociality, Symbiose and Ecology, Abe T., Bignell D. E., Higashi M. (eds). Kuwer Academic Publishers: 25-51.

13. Emerson A. E. (1928). Termites of the Belgian Congo and the Cameroon. Bull. Amer: Mus.

14. Nat. Hist. New-York, 57, 212-222.

15. Engel M. S. (2011). Family-group names for termites (Isoptera), redux. In: Engel MS (Ed) Contributions Celebrating Kumar Krishna. ZooKeys 148: 171-184. doi : 10.3897/Zookeys.148.1682. 
16. Engel M. S., Grimaldi D. A. \& Krishna K. (2009). Termites (Isoptera): Their phylogeny, classification, and rise to ecological dominance. American Museum Novitates 3650 : 1-27. doi : 10.1206/651.1.

17. Fall S., Sarr M., Rouland C., Agbogba C. \& Brauman A. (2000). Effet de l'âge de la jachère et de la saison sur la densité et la diversité des termites (Haute-Casamance, Sénégal). In : La jachère en Afrique tropicale - Ch. Floret, R. Pontanier; John Libbey Eurotext, Paris, pp. 259-267.

18. Freymann B. P., Buitenwerf R., Desouza Og. \& Olff H. (2008). The importance of termites (Isoptera) for the recycling of herbivore dung in tropical ecosystem: a review. Eur. J. Entomol. 105: 165-173. doi :10.14411/eje.2008.025.

19. Garba M., Cornelis W. M. \& Steppe K. (2011). Effet of termite mound material on the physical proprieties of sandy soil and on the growth characteristics of tomato (Solanum lycopersicum L.) in semiarid Niger. Plant Soil. 338: 451-466.

20. Grassé P.-P. (1937). Recherches sur la systématique et la biologie des Termites de l'Afrique occidentale française. Première partie, Protermtidae, Mesotermitidae, Metatermitidae, (Termitinae). Annales de la société entomologique de France, CVI, 1-104.

21. Grassé P. P. (1944-45). Recherches sur la biologie des termites champignonnistes (Macrotermitinae). Extrait des Annales Sc. Nat. Zoologie - 11 ${ }^{\mathrm{e}}$ série-1944-1945-T.VI et VII.

22. Grassé P. P. (1984). Termitologia. Tome II. Fondation des sociétés, construction. Masson, paris, 613p.

23. Guèye N. (1987). Rôle des termites dans des plantations forestières du Cap-Vert (Mbao, Sénégal). Thèse de Doctorat de l’Université Paris 6, 159p.

24. Guèye S. (2011). Contribution à l'étude de la faune de Termites (lsoptera) dans la Réserve de Faune du Ferlo Nord (Sénégal): cas de l'enclos d'acclimatation de Katane. Mémoire de Master II, Université Ch. A. Diop de Dakar, 28p.

25. Han S. H. \& Ndiaye A. B. (1996). Dégâts causés par les termites (Isoptera) sur les arbres fruitiers de la région de Dakar (Sénégal). Actes Coll. Insectes Sociaux, 10 : 111-117.

26. Han S. H. \& Ndiaye A. B. (1998). L'attaque des cultures maraîchères par les termites (Isoptera) dans la région de Dakar (Sénégal). Actes Coll. Insectes Sociaux, 11 : 37-43.

27. Han S. H. (2000). Dégâts causés par les termites sur les bâtiments dans la région de Dakar au Sénégal. Actes Coll. Insectes Sociaux, 13 : 61-64. 
28. Han S. H. \& Ndiaye A. B. (2006). L'attaque des arbres fruitiers par les termites dans la région de Thiès (Sénégal) (Isoptera). Bulletin de la société entomologique de France, 111(1) : 59-64

29. Jouquet P., Traoré S., Choosai C., Hartmann C. \& Bignell D. (2011). Influence of termites on ecosystem functioning. Ecosystem services provided by termites. European Journal of Soil Biology 47: 215-222.

30. Krishna K., Grimaldi D. A., Krishna V. \& Engel M. S. (2013). Treatise of Isoptera of the world. Bulletin of the American Museum of Natural History 377 (7 vol.) 2704p.

31. Lepage M. (1974). Les Termites d'une savane sahélienne (Ferlo septentrional, Sénégal): peuplement, population, consommation et rôle dans l'écosystème. Thèse de doctorat ès-Sciences, Université de Dijon, 344p.

32. Mbao C. D. (2015). Les peuplements de termites (Isoptera) de vergers du plateau de Diass et de la Niaye de Bayakh (Sénégal). Mémoire de Master II, Université Ch. A. Diop de Dakar, 30p.

33. Ndiaye A. B. \& Han S. H. (2002). Attaque des arbres fruitiers par les Termites en Casamance (Sénégal) (Isoptera). Bulletin de la Société entomologique de France, 107 (2) : 193-199.

34. Ndiaye A. B. \& Samb T. (2012). Les Termites (Isoptera) dans les parcelles de reboisement de la grande muraille verte entre Widou Thiengoly et Tessekere. Les Cahiers de l'Observatoire International Homme-Milieux Tessekere, 1, 63-73.

35. Ndiaye A. B. (2014). Contribution à la connaissance des Termites (Isoptera Brullé, 1832) du Sénégal: Systématique et Ecologique. Thèse de doctorat ès-Sciences, spécialité Entomologie, Université Cheikh Anta Diop de Dakar,

36. Niane A. B. (1984). Etudes cartographiques et agro-pédologiques des sols du plateau de Basse-Casamance. Mémoire de stage, Institut Sénégalais de Recherches Agricoles (ISRA). Centre de Recherches Agricoles de Djibélor, 98p.

37. Noirot Ch. \& Alliot H. (1947). La lutte contre les termites. Office de la recherche scientifique coloniale. Masson \& $\mathrm{C}^{\mathrm{ie}}$, Editeurs, 93p.

38. Ouedraogo E., Mando A. \& Brussaard L. (2004). Soil macrofaunalmediated organic resource disappearance in semi-arid West Africa. Applied Soil Ecology 27 : 259-267.

39. Roisin Y. \& Leponce M. (2004). Characterizing termite assemblages in fragmented forests: A test case in the Argentinian Chaco. Austral Ecology 29, 637-646.

40. Roy-Noël J. (1974). Recherches sur l'Écologie des Isoptères de la Presqu'ile du Cap-Vert (Sénégal). Bull. de l'IFAN, 36, A, 2-3, 291609. 
41. Roy-Noël J. (1978). Influence de l’Homme sur le peuplement en Termites de la Presqu’ile du Cap-Vert (Sénégal occidental). Memorabila Zoologica, 29, 157-172.

42. Roy-Noël J. \& Wane C. (1977). L’attaque des arbres par les Termites dans la presqu’île du Cap-Vert (Sénégal). Bulletin l’IFAN. T.39. sér. A, $n^{\circ} 1$.

43. Roy-Noël J. (1982). L’attaque des arbres par les termites dans la presqu'île du Cap-Vert (Sénégal). Bulletin de l’IFAN. T. 44. Sér.A, $n^{\circ} 1-2$.

44. Samb T., Ndiaye A. B. \& Diarra K. (2011). Biodiversity of Termites in relation to human

45. activity: impact on the environment in Matam (Senegal). Research Journal of Pharmaceutical, Biological and Chemical Sciences, 2 (1), 313-323.

46. Sarr M (1999). Étude écologique des peuplements de Termites dans les jachères et dans les cultures en zone soudano-sahélienne, au Sénégal. Thèse de doctorat de 3ème cycle de

47. Biologie animale, Université Ch. A. Diop de Dakar, 117 p.

48. Sarr M., Maniania N. K., Russel-Smith A. \& Niassy A. (2005). Diversité des termites (Isoptera) au Sénégal et leurs dégâts sur les cultures et périmètres boisés. International Journal of Tropical Insect Science. Vol. 25, No. 3, pp. 147-158. doi: 10.1079/IJT200575.

49. Seck A. (1955). La moyenne Casamance. Etude de géographie physique, In : Revue de géographie alpine. Tome 43, N 4. pp. 707755.

50. Silvestri F. (1914-15). Contribuzione alla conoscenza dei Termitidi e Termitophili dell'Africa occidentale. I. Termitidi. Bolletino del Laboratorio di Zoologia Generale e Agraria della

51. R. Scuola Superore d'Agricoltura, Portici, 9, 1-146.

52. Sjostedt Y. (1925). Revision der Termiten Afrikas. 3. Monographie. Kungl Svenska Vetenska

53. Akademiens handlingar, 3 (1), 1-435.

54. Sonko A. M. (2015). Les attaques de Termites (Isoptera, Brullé) sur le Manguier (Mangifera indica L.) dans des vergers du plateau de Diass et des Niayes de Bayakh (Sénégal). Mémoire de Master II, Université Ch. A. Diop de Dakar, 49p.

55. Tano Y. \& Lepage M. (1990). Les Termites : dynamique des nids épigés et interactions avec les composantes du milieu. https://www.researchgate.net/publication/32980105.

56. Tra Bi C. S., Konaté S. \& Tano Y. (2010). Diversité et abondance des Termites (Insecta: Isoptera) dans un gradient d’âge de paillis de 
cabosses (Oumé-Côte d’Ivoire). Journal of Animal \& Plant Sciences, 2010. Vol. 6, Issue 3: 685- 699.

57. Zaremski A., Fouquet D. \& Louppe D. (2009). Les termites dans le monde. Editions Quae, 93p.

58. Zaremski A. \& Louppe D. (2009). Les termites. Conférence donnée le 10 mai 2016 au Muséum d'histoire naturelle de Nantes à l'occasion des Mardis muséum, les rendez-vous de la science. https://agritrop.cirad.fr/580482/1/Conférence\%20termites\%20AZ\%2 0DL.docx.

Tableau I. Espèces de termites et leur distribution dans les biotopes à Médina Yoro Foula (Kolda, Sénégal)

\begin{tabular}{|c|c|c|}
\hline MEDINA YERO FOULA & Savane boisée & Rizière \\
\hline Coptotermes intermedius Silvestri, 1912 & Nid & - \\
\hline Ancistrotermes cavithorax (Sjostedt, 1899) & Bois mort & Arbuste ; Bois mort \\
\hline Macrotermes bellicosus (Smeathman, 1781) & Nid ; bois mort & - \\
\hline Macrotermes subhyalinus (Rambur, 1842) & Nid & - \\
\hline Microtermes grassei Ghidini, 1955 & $\begin{array}{l}\text { Souche ; nids; } \\
\text { arbustes ; bois } \\
\text { mort ; bouse de } \\
\text { vache }\end{array}$ & Bois mort \\
\hline Microtermes lepidus Sjöstedt, 1924 & arbre ; sol & - \\
\hline Microtermes subhyalinus Silvestri, 1914 & Bois mort & - \\
\hline Odontotermes pauperans (Silvestri, 1912) & Bouse de vache & - \\
\hline Odontotermes sp. 2 & bois mort & \\
\hline Trinervitermes geminatus (Rambur, 1842) & Nid & - \\
\hline Trinervitermes occidentalis (Sjöstedt, 1904) & - & Nid \\
\hline Trinervitermes togoensis (Sjöstedt, 1899) & Sol; souche & Nid \\
\hline Trinervitermes trinervius (Rambur, 1842) & Sous sol & Sol ; nids \\
\hline Cubitermes niokoloensis Roy-Noël, 1969 & Nid & - \\
\hline Cubitermes sp. 1 & Nid & - \\
\hline Cubitermes sp. 2 & Nid & - \\
\hline Noditermes cristifrons (Wasmann, 1911) & Nids & - \\
\hline Amitermes evuncifer (Silvestri, 1912) & Souche & - \\
\hline Microcerotermes sp.aff. parvulus (Sjostedt, 1911) & - & Arbre \\
\hline Microcerotermes spp. & Nids & Arbre \\
\hline
\end{tabular}


Tableau II. Espèces de termites et leur distribution dans les biotopes à Saré Samba Kayogo (Kolda, Sénégal)

\begin{tabular}{|c|c|c|}
\hline SARE SAMBA KAYOGO & $\begin{array}{l}\text { Savane boisée } \\
\text { (forêt classée de } \\
\text { Bacor) }\end{array}$ & $\begin{array}{c}\text { Palmeraie } \\
\text { anthropisée } \\
\text { (bord d'un cours } \\
\text { d'eau) }\end{array}$ \\
\hline Ancistrotermes cavithorax (Sjostedt, 1899) & Bois mort ; arbustes & $\begin{array}{l}\text { Arbres ; litière, } \\
\text { bois mort }\end{array}$ \\
\hline Ancistrotermes guineensis (Silvestri, 1912) & Bois mort & Bois mort \\
\hline Macrotermes bellicosus (Smeathman, 1781) & Nid ; litière & - \\
\hline Macrotermes subhyalinus (Rambur, 1842) & bois mort & Bois mort \\
\hline Microtermes grassei Ghidini, 1955 & Bois mort, sol & - \\
\hline Microtermes subhyalinus Silvestri, 1914 & litière & - \\
\hline Odontotermes erraticus Grassé 1944-45 & Bouse de vache ; arbre & - \\
\hline Odontotermes sp. 1 & $\begin{array}{c}\text { Bouse de vache ; bois } \\
\text { mort }\end{array}$ & - \\
\hline Odontotermes sp. 2 & Bois mort & - \\
\hline Trinervitermes togoensis (Sjöstedt, 1899) & - & Nid \\
\hline Cubitermes sp. 2 & - & $\begin{array}{c}\text { Sol au pied d'un } \\
\text { palmier }\end{array}$ \\
\hline Cubitermes sp. (ouvriers indét.) & Nid & - \\
\hline \multirow{5}{*}{$\begin{array}{l}\text { Amitermes evuncifer (Silvestri, 1912) } \\
\text { Microcerotermes sp.aff. parvulus (Sjostedt, 1911) } \\
\text { Microcerotermes sp.aff. solidus Silvestri, 1912 } \\
\text { Microcerotermes sp. (ouvriers indét.) } \\
\text { Promirotermes holmgreni infera Silvestri, } 1914\end{array}$} & Arbre ; nid ; souche & $\begin{array}{l}\text { Bois mort ; } \\
\text { souche }\end{array}$ \\
\hline & - & Arbre \\
\hline & - & Bois mort \\
\hline & Arbre & - \\
\hline & Nid & - \\
\hline
\end{tabular}

Tableau III. Espèces de termites et leur distribution dans les biotopes à Wassadou (Kolda, Sénégal)

\begin{tabular}{|c|c|c|c|c|}
\hline WASSADOU & $\begin{array}{c}\text { Cuvette } \\
\text { inondable }\end{array}$ & $\begin{array}{l}\text { Champs et } \\
\text { jachères }\end{array}$ & Verger & $\begin{array}{l}\begin{array}{l}\text { Savane } \\
\text { boisée }\end{array} \\
\end{array}$ \\
\hline Coptotermes intermedius Silvestri, 1912 & 一 & - & & $\begin{array}{c}\text { Arbuste; } \\
\text { bois mort }\end{array}$ \\
\hline Ancistrotermes cavithorax (Sjostedt, 1899) & $\begin{array}{c}\text { Arbres ; } \\
\text { arbustes ; } \\
\text { litière et bois } \\
\text { mort }\end{array}$ & - & $\begin{array}{l}\text { Nids; } \\
\text { bois } \\
\text { mort ; } \\
\text { arbre ; } \\
\text { souche }\end{array}$ & - \\
\hline Macrotermes bellicosus (Smeathman, 1781) & - & $\begin{array}{c}\text { Nid; bois } \\
\text { mort }\end{array}$ & & - \\
\hline Macrotermes subhyalinus (Rambur, 1842) & Bois mort & - & & - \\
\hline Microtermes grassei Ghidini, 1955 & Bois mort & Bois mort & Nid & - \\
\hline Microtermes lepidus Sjöstedt, 1924 & $\begin{array}{l}\text { Bois mort ; } \\
\text { arbre }\end{array}$ & $\begin{array}{c}\text { Bois mort ; } \\
\text { bouses de } \\
\text { vache ; } \\
\text { placages } \\
\text { au sol }\end{array}$ & & Bois mort \\
\hline Microtermes subhyalinus Silvestri, 1914 & $\begin{array}{l}\text { Souche } \\
\text { d'arbre }\end{array}$ & - & Nid & - \\
\hline Odontotermes erraticus Grassé 1944-45 & - & $\begin{array}{c}\text { Bouse de } \\
\text { vache ; }\end{array}$ & & Bois mort \\
\hline
\end{tabular}


Odontotermes sudanensis Sjostedt, 1924

\begin{tabular}{|c|c|c|c|}
\hline \multicolumn{4}{|c|}{ arbre } \\
\hline - & - & $\begin{array}{l}\text { Bouse de } \\
\text { vache ; } \\
\text { bois mort }\end{array}$ & - \\
\hline - & $\begin{array}{l}\text { Bouses de } \\
\text { vache ; } \\
\text { bois mort ; }\end{array}$ & $\begin{array}{l}\text { Bois } \\
\text { mort }\end{array}$ & - \\
\hline Bois mort & - & & - \\
\hline
\end{tabular}

\begin{tabular}{|c|c|c|c|c|}
\hline Fulleritermes tenebricus & - & - & $\begin{array}{c}\text { Arbre ; } \\
\text { nid }\end{array}$ & arbuste \\
\hline Trinervitermes trinervius (Rambur, 1842) & Nid & Nids & $\begin{array}{c}\text { Arbre ; } \\
\text { nid }\end{array}$ & - \\
\hline Cubitermes curtatus & - & - & Nids & $\begin{array}{c}\text { Souche ; } \\
\text { nid }\end{array}$ \\
\hline Cubitermes sp. 1 & - & Nid & Nid & - \\
\hline Cubitermes sp. (ouvriers indét.) & - & - & Nid & Nids \\
\hline Noditermes cristifrons (Wasmann, 1911) & - & - & $\begin{array}{l}\text { Nids; } \\
\text { arbre }\end{array}$ & - \\
\hline Amitermes evuncifer (Silvestri, 1912) & $\begin{array}{c}\text { Arbre mort ; } \\
\text { souche } \\
\text { d'arbre }\end{array}$ & - & Nid & Souche \\
\hline Microcerotermes fuscotibialis (Sjostedt, 1896) & - & - & Arbres & - \\
\hline Microcerotermes sp.aff. parvulus (Sjostedt, 1911) & - & - & & Souche \\
\hline Microcerotermes sp.aff.solidus Silvestri, 1912 & - & - & Nid & $\begin{array}{c}\text { Arbuste, } \\
\text { bois mort ; } \\
\text { nid }\end{array}$ \\
\hline Microcerotermes sp. (ouvriers indét.) & Bois mort & - & & $\begin{array}{c}\text { Bois mort ; } \\
\text { arbuste }\end{array}$ \\
\hline Pericapritermes urgens Silvestri, 1914 & - & - & Nids & - \\
\hline Tuberculitermes bycanistes Sjöstedt 1905 & - & - & Nid & - \\
\hline
\end{tabular}

Tableau IV. Composition spécifique des groupes trophiques

\begin{tabular}{|c|c|}
\hline Groupes trophiques & Espèces \\
\hline Lignivores & $\begin{array}{c}\text { Coptotermes intermedius, Amitermes evuncifer, Microcerotermes sp.aff. } \\
\text { parvulus, Microcerotermes sp. aff. solidus, M. fuscotibialis, } \\
\text { Fulleritermes tenebricus. }\end{array}$ \\
\hline HuI & $\begin{array}{c}\text { Cubitermes curtatus, C. niokoloensis, Cubitermes sp. 1, Cubitermes sp. } \\
\text { 2, Noditermes cristifrons, Tuberculitermes bycanistes, Pericapritermes } \\
\text { urgens, Promirotermes holmgreni infera. }\end{array}$ \\
\hline Champignonnistes & $\begin{array}{l}\text { Ancistrotermes cavithorax, A. guineensis, Macrotermes bellicosus, } \\
\text { Macrotermes subhyalinus, Microtermes grassei, } \text { M. lepidus, } \\
\text { Microtermes subhyalinus, Odontotermes erraticus, O. pauperans, O. } \\
\text { sudanensis, Odontotermes sp. 1, Odontotermes sp. } 2 .\end{array}$ \\
\hline Fourrageu & gensis, T. geminatus, T. occidentalis \\
\hline
\end{tabular}




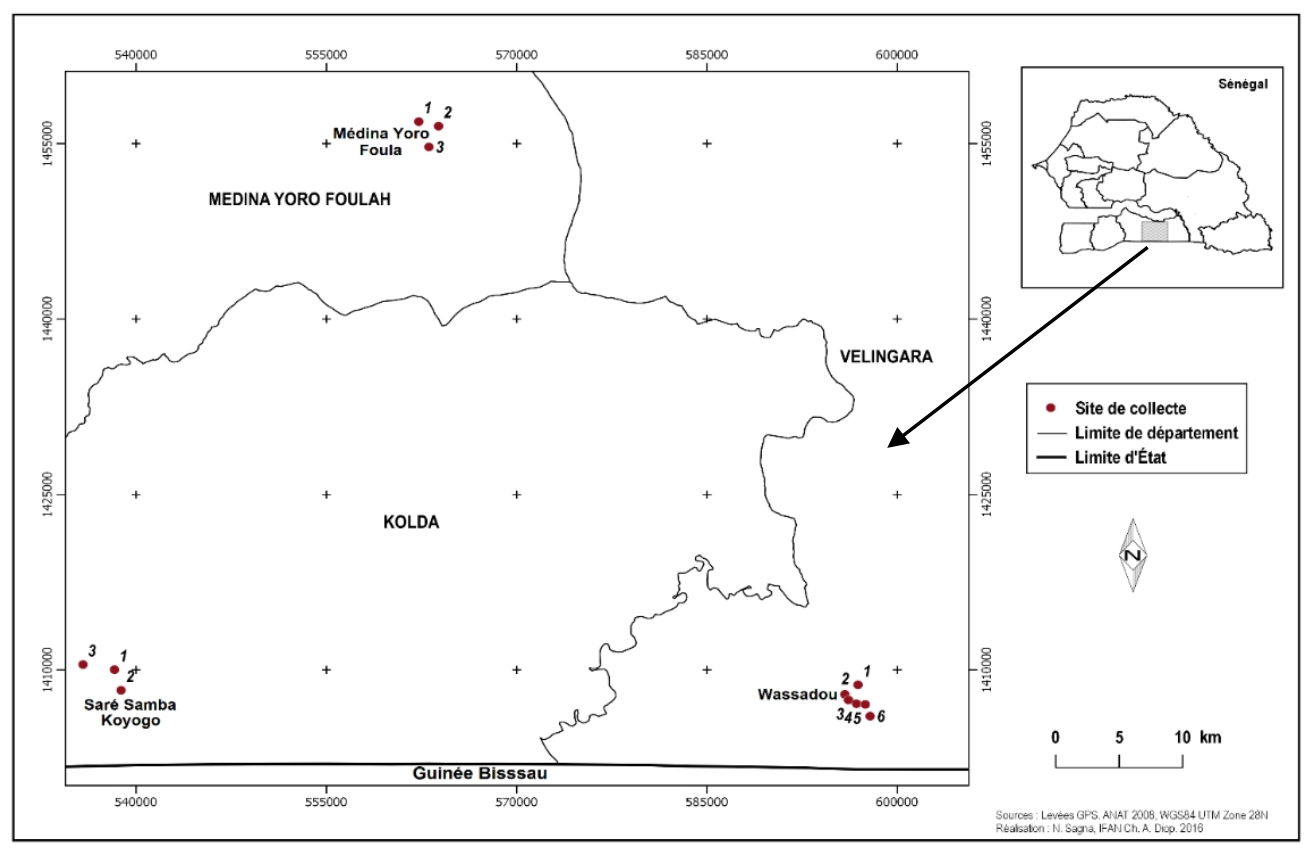

Figure 1. Localisation des sites d’étude

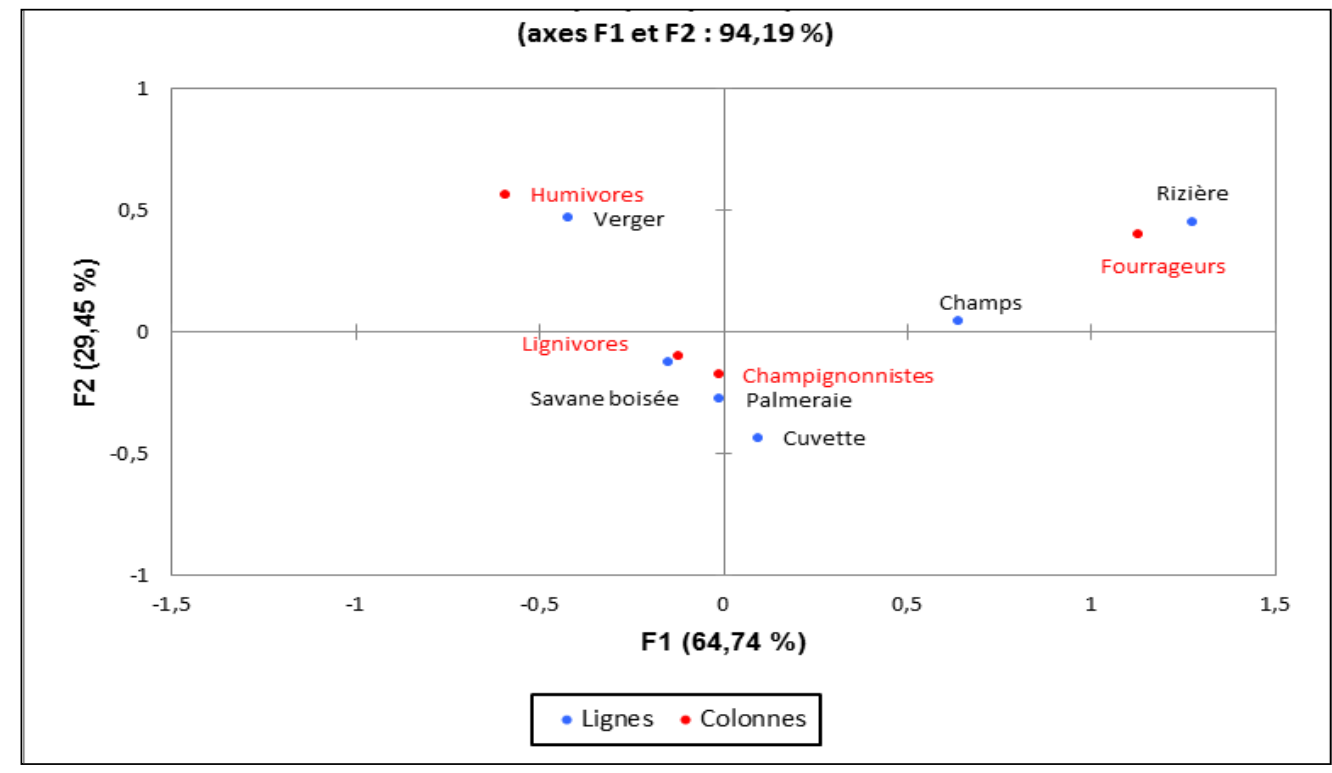

Fig. 2.Répartition des groupes trophiques selon les biotopes 


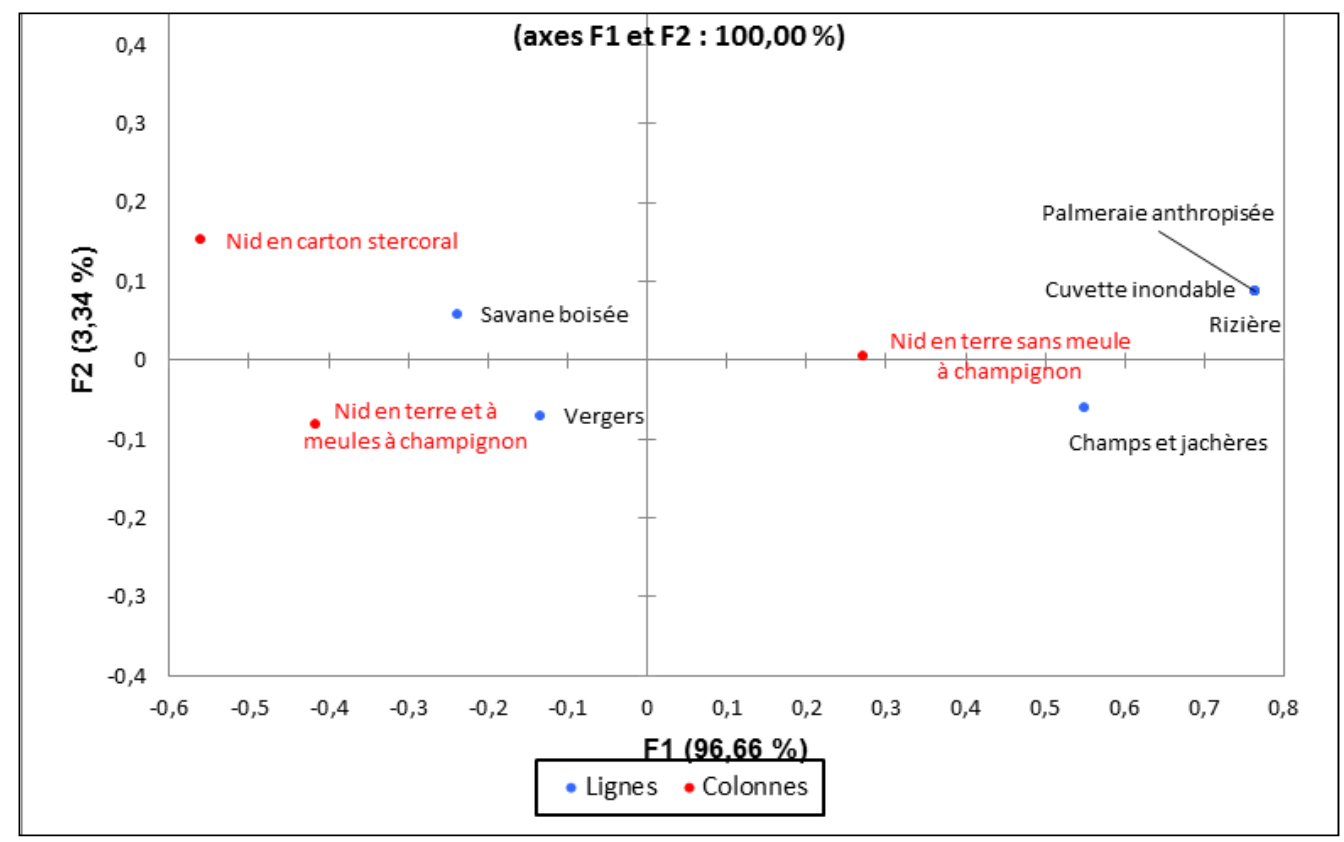

Fig.3. Répartition des types de nids selon les biotopes 